\title{
Time Period Identification of Events in Text
}

\author{
Taichi Noro $^{\dagger} \quad$ Takashi Inui $^{\dagger \dagger} \quad$ Hiroya Takamura $^{\ddagger} \quad$ Manabu Okumura $^{\ddagger}$ \\ ${ }^{\dagger}$ Interdisciplinary Graduate School of Science and Engineering \\ Tokyo Institute of Technology \\ 4259 Nagatsuta-cho, Midori-ku, Yokohama, Kanagawa, Japan \\ ${ }^{\dagger+} J a p a n$ Society for the Promotion of Science \\ ${ }^{\ddagger}$ Precision and Intelligence Laboratory, Tokyo Institute of Technology \\ \{norot, tinui\}@lr.pi.titech.ac.jp, \{takamura, oku\}@pi.titech.ac.jp
}

\begin{abstract}
This study aims at identifying when an event written in text occurs. In particular, we classify a sentence for an event into four time-slots; morning, daytime, evening, and night. To realize our goal, we focus on expressions associated with time-slot (time-associated words). However, listing up all the time-associated words is impractical, because there are numerous time-associated expressions. We therefore use a semi-supervised learning method, the Naïve Bayes classifier backed up with the Expectation Maximization algorithm, in order to iteratively extract time-associated words while improving the classifier. We also propose to use Support Vector Machines to filter out noisy instances that indicates no specific time period. As a result of experiments, the proposed method achieved 0.864 of accuracy and outperformed other methods.
\end{abstract}

\section{Introduction}

In recent years, the spread of the internet has accelerated. The documents on the internet have increased their importance as targets of business marketing. Such circumstances have evoked many studies on information extraction from text especially on the internet, such as sentiment analysis and extraction of location information. In this paper, we focus on the extraction of temporal information. Many authors of documents on the web often write about events in their daily life. Identifying when the events occur provides us valuable information. For example, we can use temporal information as a new axis in the information retrieval. From time-annotated text, companies can figure out when customers use their products. We can explore activities of users for marketing researches, such as "What do people eat in the morning?", "What do people spend money for in daytime?”

Most of previous work on temporal processing of events in text dealt with only newswire text. In those researches, it is assumed that temporal expressions indicating the time-period of events are often explicitly written in text. Some examples of explicit temporal expressions are as follows: "on March 23”, “at 7 p.m.”.

However, other types of text including web diaries and blogs contain few explicit temporal expressions. Therefore one cannot acquire sufficient temporal information using existing methods. Although dealing with such text as web diaries and blogs is a hard problem, those types of text are excellent information sources due to their overwhelmingly huge amount.

In this paper, we propose a method for estimating occurrence time of events expressed in informal text. In particular, we classify sentences in text into one of four time-slots; morning, daytime, evening, and night. To realize our goal, we focus on expressions associated with time-slot (hereafter, called time-associated words), such as "commute (morning)", "nap (daytime)" and "cocktail (night)". Explicit temporal expressions have more certain information than the timeassociated words. However, these expressions are rare in usual text. On the other hand, although the time-associated words provide us only indirect information for estimating occurrence time of events, these words frequently appear in usual text. Actually, Figure 2 (we will discuss the graph in Section 5.2, again) shows the number of sentences including explicit tem- 
poral expressions and time-associated words respectively in text. The numbers are obtained from a corpus we used in this paper. We can figure out that there are much more time-associated words than explicit temporal expressions in blog text. In other words, we can deal with wide coverage of sentences in informal text by our method with time-associated words.

However, listing up all the time-associated words is impractical, because there are numerous time-associated expressions. Therefore, we use a semi-supervised method with a small amount of labeled data and a large amount of unlabeled data, because to prepare a large quantity of labeled data is costly, while unlabeled data is easy to obtain. Specifically, we adopt the Naïve Bayes classifier backed up with the Expectation Maximization (EM) algorithm (Dempster et al., 1977) for semi-supervised learning. In addition, we propose to use Support Vector Machines to filter out noisy sentences that degrade the performance of the semi-supervised method.

In our experiments using blog data, we obtained 0.864 of accuracy, and we have shown effectiveness of the proposed method.

This paper is organized as follows. In Section 2 we briefly describe related work. In Section 3 we describe the details of our corpus. The proposed method is presented in Section 4. In Section 5, we describe experimental results and discussions. We conclude the paper in Section 6.

\section{Related Work}

The task of time period identification is new and has not been explored much to date.

Setzer et al. (2001) and Mani et al. (2000) aimed at annotating newswire text for analyzing temporal information. However, these previous work are different from ours, because these work only dealt with newswire text including a lot of explicit temporal expressions.

Tsuchiya et al. (2005) pursued a similar goal as ours. They manually prepared a dictionary with temporal information. They use the handcrafted dictionary and some inference rules to determine the time periods of events. In contrast, we do not resort to such a hand-crafted material, which requires much labor and cost. Our method automatically acquires temporal information from actual data of people's activities (blog). Henceforth, we can get temporal information associated with your daily life that would be not existed in a dictionary.

\section{Corpus}

In this section, we describe a corpus made from blog entries. The corpus is used for training and test data of machine learning methods mentioned in Section 4.

The blog entries we used are collected by the method of Nanno et al. (2004). All the entries are written in Japanese. All the entries are split into sentences automatically by some heuristic rules. In the next section, we are going to explain "time-slot" tag added at every sentence.

\subsection{Time-Slot Tag}

The "time-slot" tag represents when an event occurs in five classes; "morning", "daytime", "evening", "night”, and "time-unknown". "Timeunknown" means that there is no temporal information. We set the criteria of time-slot tags as follows.

Morning: 04:00--10:59

from early morning till before noon, breakfast

Daytime: 11:00--15:59

from noon till before dusk, lunch

Evening: 16:00--17:59

from dusk till before sunset

Night: 18:00--03:59

from sunset till dawn, dinner

Note that above criteria are just interpreted as rough standards. We think time-slot recognized by authors is more important. For example, in a case of "about 3 o'clock this morning" we judge the case as "morning" (not "night") with the expression written by the author "this morning".

To annotate sentences in text, we used two different clues. One is the explicit temporal expressions or time-associated words included in the sentence to be judged. The other is contextual information around the sentences to be judged. The examples corresponding to the former case are as follows:

\section{Example 1}

a. I went to post office by bicycle in the morning. b. I had spaghetti at restaurant at noon.

c. I cooked stew as dinner on that day.

Suppose that the two sentences in Example 2 appear successively in a document. In this case, we first judge the first sentence as morning. Next, we judge the second sentence as morning by contextual information (i.e., the preceding sentence is judged as morning), although we cannot know the time period just from the content of the second sentence itself. 


\section{Example 2}

1. I went to $\mathrm{X}$ by bicycle in the morning.

2. I went to a shop on the way back from $X$.

\subsection{Corpus Statistics}

We manually annotated the corpus. The number of the blog entries is 7,413 . The number of sentences is 70,775 . Of 70,775 , the number of sentences representing any events ${ }^{1}$ is 14,220 . The frequency distribution of time-slot tags is shown in Table 1. We can figure out that the number of time-unknown sentences is much larger than the other sentences from this table. This bias would affect our classification process. Therefore, we propose a method for tackling the problem.

\begin{tabular}{cr}
\hline \hline morning & 711 \\
daytime & 599 \\
evening & 207 \\
night & 1,035 \\
time-unknown & 11,668 \\
\hline Total & 14,220 \\
\hline
\end{tabular}

Table 1: The numbers of time-slot tags.

\section{Proposed Method}

\subsection{Basic Idea}

Suppose, for example, "breakfast" is a strong clue for the morning class, i.e. the word is a time-associated word of morning. Thereby we can classify the sentence "I have cereal for breakfast." into the morning class. Then "cereal" will be a time-associated word of morning. Therefore we can use "cereal" as a clue of timeslot classification. By iterating this process, we can obtain a lot of time-associated words with bootstrapping method, improving sentence classification performance at the same time.

To realize the bootstrapping method, we use the EM algorithm. This algorithm has a theoretical base of likelihood maximization of incomplete data and can enhance supervised learning methods. We specifically adopted the combination of the Naïve Bayes classifier and the EM algorithm. This combination has been proven to be effective in the text classification (Nigam et al., 2000).

\footnotetext{
${ }^{1}$ The aim of this study is time-slot classification of events. Therefore we treat only sentences expressing an event.
}

\subsection{Naïve Bayes Classifier}

In this section, we describe multinomial model that is a kind of Naïve Bayes classifiers.

A generative probability of example $x$ given a category $c$ has the form:

$$
P(x \mid c, \theta)=P(|x|)|x| ! \prod_{w} \frac{P(w \mid c)^{N(w, x)}}{N(w, x)}
$$

where $P(|x|)$ denotes the probability that a sentence of length $|x|$ occurs, $N(w, x)$ denotes the number of occurrences of $w$ in text $x$. The occurrence of a sentence is modeled as a set of trials, in which a word is drawn from the whole vocabulary.

In time-slot classification, the $x$ is correspond to each sentence, the $C$ is correspond to one of time-slots in \{morning, daytime, evening, night . Features are words in the sentence. A detailed description of features will be described in Section 4.5 .

\subsection{Incorporation of Unlabeled Data with the EM Algorithm}

The EM algorithm (Dempster et al., 1977) is a method to estimate a model that has the maximal likelihood of the data when some variables cannot be observed (these variables are called latent variables). Nigam et al. (2000) proposed a combination of the Naïve Bayes classifiers and the EM algorithm.

Ignoring the unrelated factors of Eq. (1), we obtain

$$
\begin{aligned}
& P(x \mid c, \theta) \propto \prod_{w} P(w \mid c)^{N(w, x)}, \quad(2) \\
& P(x \mid \theta) \propto \sum_{c} P(c) \prod_{w} P(w \mid c)^{N(w, x)} .
\end{aligned}
$$

We express model parameters as $\theta$.

If we regard $c$ as a latent variable and introduce a Dirichlet distribution as the prior distribution for the parameters, the Q-function (i.e., the expected log-likelihood) of this model is defined as:

$$
\begin{aligned}
& Q(\theta \mid \bar{\theta})=\log (P(\theta))+\sum_{x \in D} \sum_{c} P(x \mid c, \bar{\theta}) \times \\
& \log \left(P(c) \prod_{w} P(w \mid c)^{N(w, x)},\right.
\end{aligned}
$$

where $P(\theta) \propto \prod_{c}\left(P(c)^{\alpha-1} \prod_{w}\left(P(w \mid c)^{\alpha-1}\right)\right) \cdot \alpha$ is a user given parameter and $D$ is the set of examples used for model estimation.

We obtain the next EM equation from this Qfunction: 


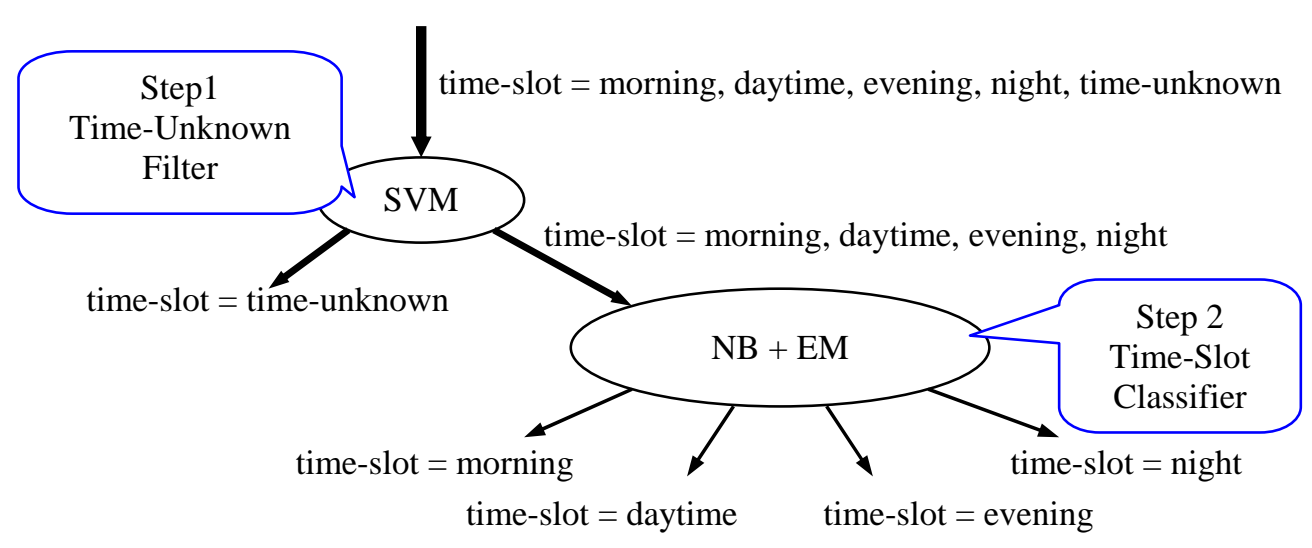

Figure 1: The flow of 2-step classification.

E-step:

$$
P(c \mid x, \bar{\theta})=\frac{P(c \mid \bar{\theta}) P(x \mid c, \bar{\theta})}{\sum_{c} P(c \mid \bar{\theta}) P(x \mid c, \bar{\theta})},,
$$

M-step:

$$
\begin{aligned}
& P(c)=\frac{(\alpha-1)+\sum_{x \in D} P(c \mid x, \bar{\theta})}{(\alpha-1)|C|+|D|},(6) \\
& P(w \mid c) \\
& =\frac{(\alpha-1)+\sum_{x \in D} P(c \mid x, \bar{\theta}) N(w, x)}{(\alpha-1)|w|+\sum_{w} \sum_{x \in D} P(c \mid x, \bar{\theta}) N(w, x)},
\end{aligned}
$$

where $|C|$ denotes the number of categories, $|W|$ denotes the number of features variety. For labeled example $x$, Eq. (5) is not used. Instead, $P(c \mid x, \bar{\theta})$ is set as 1.0 if $c$ is the category of $x$, otherwise 0 .

Instead of the usual EM algorithm, we use the tempered EM algorithm (Hofmann, 2001). This algorithm allows coordinating complexity of the model. We can realize this algorithm by substituting the next equation for Eq. (5) at E-step:

$$
P(c \mid x, \bar{\theta})=\frac{\{P(c \mid \bar{\theta}) P(x \mid c, \bar{\theta})\}^{\beta}}{\sum_{c}\{P(c \mid \bar{\theta}) P(x \mid c, \bar{\theta})\}^{\beta}},
$$

where $\beta$ denotes a hyper parameter for coordinating complexity of the model, and it is positive value. By decreasing this hyper-parameter $\beta$, we can reduce the influence of intermediate classification results if those results are unreliable.

Too much influence by unlabeled data sometimes deteriorates the model estimation. Therefore, we introduce a new hyper-parameter $\lambda(0 \leq \lambda \leq 1)$ which acts as weight on unlabeled data. We exchange the second term in the righthand-side of Eq. (4) for the next equation:

$$
\begin{aligned}
& \sum_{x \in D^{\prime}} \sum_{c} P(c \mid x, \bar{\theta}) \log \left(P(c) \prod_{w} P(w \mid c)^{N(w, x)}\right) \\
& +\lambda \sum_{x \in D^{u}} \sum_{c} P(c \mid x, \bar{\theta}) \log \left(P(c) \prod_{w} P(w \mid c)^{N(w, x)}\right),
\end{aligned}
$$

where $D^{l}$ denotes labeled data, $D^{u}$ denotes unlabeled data. We can reduce the influence of unlabeled data by decreasing the value of $\lambda$.

We derived new update rules from this new Qfunction. The EM computation stops when the difference in values of the Q-function is smaller than a threshold.

\subsection{Class Imbalance Problem}

We have two problems with respect to "timeunknown" tag.

The first problem is the class imbalance problem (Japkowicz 2000). The number of timeunknown time-slot sentences is much larger than that of the other sentences as shown in Table 1. There are more than ten times as many timeunknown time-slot sentences as the other sentences.

Second, there are no time-associated words in the sentences categorized into "time-unknown". Thus the feature distribution of time-unknown time-slot sentences is remarkably different from the others. It would be expected that they adversely affect proposed method.

There have been some methodologies in order to solve the class imbalance problem, such as Zhang and Mani (2003), Fan et al. (1999) and Abe et al. (2004). However, in our case, we have to resolve the latter problem in addition to the class imbalance problem. To deal with two problems above simultaneously and precisely, we develop a cascaded classification procedure. 


\subsection{Time-Slot Classification Method}

It's desirable to treat only "time-known" sentences at NB+EM process to avoid the abovementioned problems. We prepare another classifier for filtering time-unknown sentences before NB+EM process for that purpose. Thus, we propose a classification method in 2 steps (Method A). The flow of the 2-step classification is shown in Figure 1. In this figure, ovals represent classifiers, and arrows represent flow of data.

The first classifier (hereafter, "time-unknown" filter) classifies sentences into two classes; "time-unknown" and "time-known". The "timeknown" class is a coarse class consisting of four time-slots (morning, daytime, evening, and night). We use Support Vector Machines as a classifier. The features we used are all words included in the sentence to be classified.

The second classifier (time-slot classifier) classifies "time-known" sentences into four classes. We use Naïve Bayes classifier backed up with the Expectation Maximization (EM) algorithm mentioned in Section 4.3.

The features for the time-slot classifier are words, whose part of speech is noun or verb. The set of these features are called NORMAL in the rest of this paper. In addition, we use information from the previous and the following sentences in the blog entry. The words included in such sentences are also used as features. The set of these features are called CONTEXT. The features in CONTEXT would be effective for estimating time-slot of the sentences as mentioned in Example2 in Section 3.1.

We also use a simple classifier (Method B) for comparison. The Method B classifies all timeslots (morning night, time-unknown) sentences at just one step. We use Naïve Bayes classifier backed up with the Expectation Maximization (EM) algorithm at this learning. The features are words (whose part-of-speech is noun or verb) included in the sentence to be classified.

\section{Experimental Results and Discussion}

\subsection{Time-Slot Classifier with Time- Associated Words}

\subsubsection{Time-Unknown Filter}

We used 11.668 positive (time-unknown) samples and 2,552 negative (morning night) samples. We conducted a classification experiment by Support Vector Machines with 10-fold cross validation. We used TinySVM ${ }^{2}$ software package for implementation. The soft margin parameter is automatically estimated by 10 -fold cross validation with training data. The result is shown in Table 2.

Table 2 clarified that the "time-unknown" filter achieved good performance; F-measure of 0.899 . In addition, since we obtained a high recall (0.969), many of the noisy sentences will be filtered out at this step and the classifier of the second step is likely to perform well.

\begin{tabular}{cc}
\hline \hline Accuracy & 0.878 \\
Precision & 0.838 \\
Recall & 0.969 \\
F-measure & 0.899 \\
\hline
\end{tabular}

Table 2: Classification result of the time-unknown filter.

\subsubsection{Time-Slot Classification}

In step 2, we used "time-known" sentences classified by the unknown filter as test data. We conducted a classification experiment by Naïve Bayes classifier + the EM algorithm with 10-fold cross validation. For unlabeled data, we used 64,782 sentences, which have no intersection with the labeled data. The parameters, $\lambda$ and $\beta$, are automatically estimated by 10 -fold cross validation with training data. The result is shown in Table 3.

\begin{tabular}{l|c|c}
\hline \multirow{2}{*}{ Method } & \multicolumn{2}{|c}{ Accuracy } \\
\cline { 2 - 3 } & NORMAL & CONTEXT \\
\hline Explicit & \multicolumn{2}{|c}{0.109} \\
Baseline & \multicolumn{2}{|c}{0.406} \\
\hline NB & 0.567 & 0.464 \\
NB + EM & 0.673 & 0.670 \\
\hline
\end{tabular}

Table 3: The result of time-slot classifier.

\footnotetext{
${ }^{2}$ http://www.chasen.org/ taku/software/TinySVM
} 


\begin{tabular}{|c|c|c|c|c|c|c|c|}
\hline & \multicolumn{5}{|c|}{ output of time-slot classifier } & \multirow{2}{*}{ sum } \\
\hline & & morning & daytime & evening & night & time-unknown & \\
\hline \multirow{5}{*}{ త్రే } & morning & 332 & 14 & 1 & 37 & 327 & 711 \\
\hline & daytime & 30 & 212 & 1 & 44 & 312 & 599 \\
\hline & evening & 4 & 5 & 70 & 18 & 110 & 207 \\
\hline & night & 21 & 19 & 4 & 382 & 609 & 1035 \\
\hline & time-unknown & 85 & 66 & 13 & 203 & 11301 & 11668 \\
\hline & sum & 472 & 316 & 89 & 684 & 12659 & 14220 \\
\hline
\end{tabular}

Table 4: Confusion matrix of output.

\begin{tabular}{ccccccccr}
\hline \hline & morning & \multicolumn{2}{c}{ daytime } & \multicolumn{2}{c}{ evening } & night \\
\hline rank & word & $p(c \mid w)$ & word & $p(c \mid w)$ & word & $p(c \mid w)$ & word & $p(c \mid w)$ \\
\hline 1 & this morning & 0.729 & noon & 0.728 & evening & 0.750 & last night & 0.702 \\
2 & morning & 0.673 & early after noon & 0.674 & sunset & 0.557 & night & 0.689 \\
3 & breakfast & 0.659 & afternoon & 0.667 & academy & 0.448 & fireworks & 0.688 \\
4 & early morning & 0.656 & daytime & 0.655 & dusk & 0.430 & dinner & 0.684 \\
5 & before noon & 0.617 & lunch & 0.653 & Hills & 0.429 & go to bed & 0.664 \\
6 & compacted snow & 0.603 & lunch & 0.636 & run on & 0.429 & night & 0.641 \\
7 & commute & 0.561 & lunch break & 0.629 & directions & 0.429 & bow & 0.634 \\
8 & --- & 0.541 & lunch & 0.607 & pinecone & 0.429 & overtime & 0.606 \\
9 & parade & 0.540 & noon & 0.567 & priest & 0.428 & year-end party & 0.603 \\
10 & wake up & 0.520 & butterfly & 0.558 & sand beach & 0.428 & dinner & 0.574 \\
11 & leave harbor & 0.504 & Chinese food & 0.554 & --- & 0.413 & beach & 0.572 \\
12 & rise late & 0.504 & forenoon & 0.541 & Omori & 0.413 & cocktail & 0.570 \\
13 & cargo work & 0.504 & breast-feeding & 0.536 & fan & 0.413 & me & 0.562 \\
14 & alarm clock & 0.497 & nap & 0.521 & Haneda & 0.412 & Tomoyuki & 0.560 \\
15 & --- & 0.494 & diaper & 0.511 & preview & 0.402 & return home & 0.557 \\
16 & sunglow & 0.490 & Japanese food & 0.502 & cloud & 0.396 & close & 0.555 \\
17 & wheel & 0.479 & star festival & 0.502 & Dominus & 0.392 & stay up late & 0.551 \\
18 & wake up & 0.477 & hot noodle & 0.502 & slip & 0.392 & tonight & 0.549 \\
19 & perm & 0.474 & pharmacy & 0.477 & tasting & 0.391 & night & 0.534 \\
20 & morning paper & 0.470 & noodle & 0.476 & nest & 0.386 & every night & 0.521 \\
\hline
\end{tabular}

Table 5: Time-associated words examples.

In Table 3, "Explicit" indicates the result by a simple classifier based on regular expressions ${ }^{3}$ including explicit temporal expressions. The baseline method classifies all sentences into night because the number of night sentences is the largest. The "CONTEXT" column shows the results obtained by classifiers learned with the features in CONTEXT in addition to the features

\footnotetext{
${ }^{3}$ For example, we classify sentences matching following regular expressions into morning class:

[(gozen)(gozen- no)(asa) (asa- no)(am)(AM)(amno)(AM-no)][456789(10)] j i , [(04)(05)(06)(07)(08) (09)]j i , [(04)(05)(06)(07) (08) (09)]:[0-9]\{2,2\}, [456789(10)][(am)(AM)].

("gozen”, "gozen - no" means before noon. "asa”, “asa- no” means morning. “j i ” means o'clock.)
}

in NORMAL. The accuracy of the Explicit method is lower than the baseline. This means existing methods based on explicit temporal expressions cannot work well in blog text. The accuracy of the method 'NB' exceeds that of the baseline by $16 \%$. Furthermore, the accuracy of the proposed method 'NB+EM' exceeds that of the 'NB' by $11 \%$. Thus, we figure out that using unlabeled data improves the performance of our time-slot classification.

In this experiment, unfortunately, CONTEXT only deteriorated the accuracy. The time-slot tags of the sentences preceding or following the target sentence may still provide information to improve the accuracy. Thus, we tried a sequential tagging method for sentences, in which tags are 
predicted in the order of their occurrence. The predicted tags are used as features in the prediction of the next tag. This type of sequential tagging method regard as a chunking procedure (Kudo and Matsumoto, 2000) at sentence level. We conducted time-slot (five classes) classification experiment, and tried forward tagging and backward tagging, with several window sizes. We used YamCha ${ }^{4}$, the multi-purpose text chunker using Support Vector Machines, as an experimental tool. However, any tagging direction and window sizes did not improve the performance of classification. Although a chunking method has possibility of correctly classifying a sequence of text units, it can be adversely biased by the preceding or the following tag. The sentences in blog used in our experiments would not have a very clear tendency in order of tags. This is why the chunking-method failed to improve the performance in this task. We would like to try other bias-free methods such as Conditional Random Fields (Lafferty et al., 2001) for future work.

\subsubsection{2-step Classification}

Finally, we show an accuracy of the 2-step classifier (Method A) and compare it with those of other classifiers in Table 6. The accuracies are calculated with the equation:

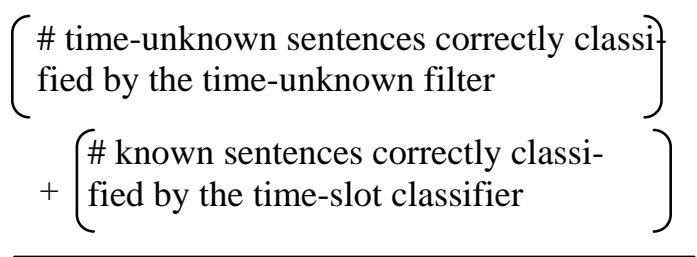

\# sentences with a time-slot tag value

In Table 6, the baseline method classifies all sentences into time-unknown because the number of time-unknown sentences is the largest. Accuracy of Method A (proposed method) is higher than that of Method B (4.1\% over). These results show that time-unknown sentences adversely affect the classifier learning, and 2-step classification is an effective method.

Table 4 shows the confusion matrix corresponding to the Method A (NORMAL). From this table, we can see Method A works well for classification of morning, daytime, evening, and night, but has some difficulty in

\footnotetext{
${ }^{4}$ http://www.chasen.org/ taku/software/YamCha
}

\begin{tabular}{cc}
\hline \hline Method & Conclusive accuracy \\
\hline Explicit & 0.833 \\
Baseline & 0.821 \\
Method A (NORMAL) & 0.864 \\
Method A (CONTEXT) & 0.862 \\
Method B & 0.823 \\
\hline
\end{tabular}

Table 6: Comparison of the methods for five class classification

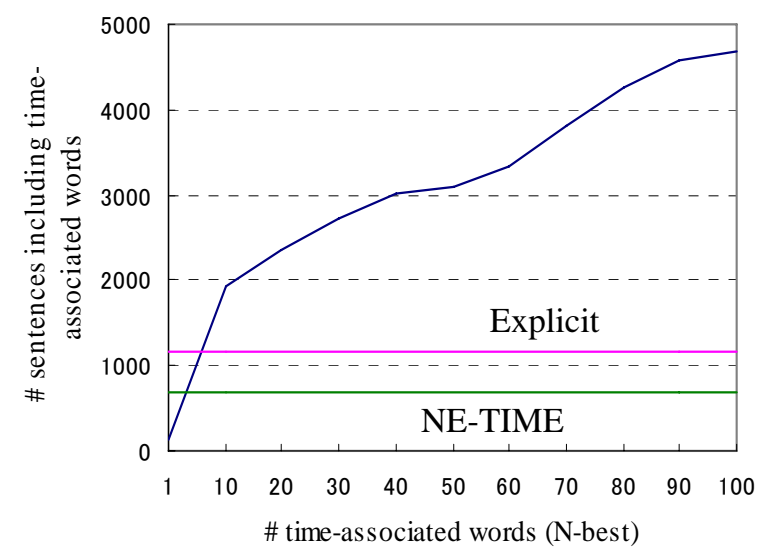

Figure 2: Change of \# sentences that have timeassociated words: "Explicit" indicates the number of sentences including explicit temporal expressions, "NE-TIME" indicates the number of sentences including NE-TIME tag.

classification of time-unknown. The $11.7 \%$ of samples were wrongly classified into "night" or "unknown".

We briefly describe an error analysis. We found that our classifier tends to wrongly classify samples in which two or more events are written in a sentence. The followings are examples:

\section{Example 3}

a. I attended a party last night, and I got back on the first train in this morning because the party was running over.

b. I bought a cake this morning, and ate it after the dinner.

\subsection{Examples of Time-Associated Words}

Table 5 shows some time-associated words obtained by the proposed method. The words are sorted in the descending order of the value of $P(c \mid w)$. Although some consist of two or three words, their original forms in Japanese consist of one word. There are some expressions appearing more than once, such as "dinner". Actually these expressions have different forms in Japanese. Meaningless (non-word) strings caused by mor- 
phological analysis error are presented as the symbol “---". We obtained a lot of interesting time-associated words, such as "commute (morning)", "fireworks (night)", and "cocktail (night)". Most words obtained are significantly different from explicit temporal expressions and NETIME expressions.

Figure 2 shows the number of sentences including time-associated words in blog text. The horizontal axis represents the number of timeassociated words. We sort the words in the descending order of $P(c \mid w)$ and selected the top $\mathrm{N}$ words. The vertical axis represents the number of sentences including any N-best time-associated words. We also show the number of sentences including explicit temporal expressions, and the number of sentences including NE-TIME tag (Sekine and Isahara, 1999) for comparison. The set of explicit temporal expressions was extracted by the method described in Section 5.1.2. We used a Japanese linguistic analyzer "Cabo$\mathrm{Cha}^{5}$ " to obtain NE-TIME information. From this graph, we can confirm that the number of target sentences of our proposed method is larger than that of existing methods.

\section{Conclusion}

In our study, we proposed a method for identifying when an event in text occurs. We succeeded in using a semi-supervised method, the Naïve Bayes Classifier enhanced by the EM algorithm, with a small amount of labeled data and a large amount of unlabeled data. In order to avoid the class imbalance problem, we used a 2-step classifier, which first filters out time-unknown sentences and then classifies the remaining sentences into one of 4 classes. The proposed method outperformed the simple 1-step method. We obtained $86.4 \%$ of accuracy that exceeds the existing method and the baseline method.

\section{References}

Naoki Abe, Bianca Zadrozny, John Langford. 2004. An Iterative Method for Multi-class Cost-sensitive Learning. In Proc. of the $10^{\text {th }}$. ACM SIGKDD, pp.3-11.

Arthur P. Dempster, Nan M. laird, and Donald B. Rubin. 1977. Maximum likelihood from incomplete data via the EM algorithm. Journal of the
Royal Statistical Society Series B, Vol. 39, No. 1, pp.1-38.

Wei Fan, Salvatore J. Stolfo, Junxin Zhang, Philip K. Chan. 1999. AdaCost: Misclassification Costsensitive Boosting. In Proc. of ICML, pp.97-105.

Thomas Hofmann. 2001. Unsupervised learning by probabilistic latent semantic analysis. Machine Learning, 42:177-196.

Nathalie Japkowicz. 2000. Learning from Imbalanced Data Sets: A Comparison of Various Strategies. In Proc. of the AAAI Workshop on Learning from Imbalanced Data Sets, pp.10 -15.

Taku Kudo, Yuji Matsumoto. 2000. Use of Support Vector Learning for Chunking Identification, In Proc of the 4th CoNLL, pp.142-144.

John Lafferty, Andrew McCallum, and Fernando Pereira. 2001. Conditional random fields: Probabilistic models for segmenting and labeling sequence data, In Proc. of ICML, pp.282-289.

Inderjeet Mani, George Wilson 2000. Robust Temporal Processing of News. In Proc. of the 38th ACL, pp.69-76.

Tomoyuki Nanno, Yasuhiro Suzuki, Toshiaki Fujiki, Manabu Okumura. 2004. Automatically Collecting and Monitoring Japanese Weblogs. Journal for Japanese Society for Artificial Intelligence , Vol.19, No.6, pp.511-520. (in Japanese)

Kamal Nigam, Andrew McCallum, Sebastian Thrun, and Tom Mitchell. 2000. Text classification from labeled and unlabeled documents using EM. Machine Learning, Vol. 39, No.2/3, pp.103-134.

Satoshi Sekine, Hitoshi Isahara. 1999. IREX project overview. Proceedings of the IREX Workshop.

Andrea Setzer, Robert Gaizauskas. 2001. A Pilot Study on Annotating Temporal Relations in Text. In Proc. of the ACL-2001 Workshop on Temporal and Spatial Information Processing, Toulose, France, July, pp.88-95.

Seiji Tsuchiya, Hirokazu Watabe, Tsukasa Kawaoka. 2005. Evaluation of a Time Judgement Technique Based on an Association Mechanism. IPSG SIG Technical Reports, 2005-NL-168, pp.113-118. (in Japanese)

Jianping Zhang, Inderjeet Mani. 2003. kNN Approach to Unbalanced Data Distributions: A Case Study involving Information Extraction. In Proc. of ICML Workshop on Learning from Imbalanced Datasets II., pp.42-48.

\footnotetext{
${ }^{5}$ http://chasen.org/ taku/software/cabocha/
} 